\title{
Development and Evaluation of Scoring Support System in Writing Test for Subject Teachers and Classroom Teachers
}

\author{
Satoshi Nakagawa*, Kazunori Sato**, Ryo Saito* and Tatsuya Horita* \\ (The original Japanese article was published in Japan Journal of Educational Technology, Vol. 43, No. 4, \\ 2020, pp. 433-445.)
}

\begin{abstract}
The purpose of this study is to develop and evaluate a system to support the scoring of writing tests in primary and secondary schools employing the subject-teacher and the classroom-teacher systems. To this end, we employ the previously proposed image-skewered method, in addition to an area setting package function, to address commercially available tests used by classroom teachers and a multiple class selection function to score tests completed by students from multiple classes taught by the same subject teacher. Further, a function is added to operate only in the case of incorrect answers to reduce the number of operations required to assess the correctness of answers. To evaluate the system, the time taken to manually score actual test papers was compared with the time taken to do so using the proposed system. The automatic scoring method exhibited a time reduction by $44.0 \%$ for subject teachers and $42.9 \%$ for classroom teachers. These results indicate that the system functions effectively as a scoring support system to reduce the time consumed for scoring tasks for teachers working in primary and secondary schools.
\end{abstract}

Keywords education software development, scoring system, writing test, primary and secondary school, reducing teacher's burden

\section{Research Issue}

\subsection{Background}

In recent years, the increased burden on primary and secondary school teachers caused by long working hours has garnered significant public attention, prompting the Ministry of Education, Culture, Sports, Science and Technology (MEXT) to issue a notice entitled "Exhaustive Measures for Work-Style Reform in Schools" ${ }^{\text {[1] }}$ to local governments and boards of education across Japan. According to a MEXT survey ${ }^{[2]}$, among the 25 duties to which teachers dedicate their time on an average weekday, besides "teaching classes," junior high school teachers spend the most amount of time on "lesson preparation" (86 minutes) and "club activities" (41 minutes), followed by "grade processing" (38 minutes). On the other hand, primary school teachers spend the most amount of time on "lesson preparation" (77 minutes) and "morning duties" (35 minutes), followed by "grade processing" (33 minutes). Therefore, the duties that consume the highest amount of time among teachers at both primary and junior high schools are observed to be lesson preparation and grade processing.

Reports have established that the use of

\footnotetext{
* Graduate School of Information Sciences, Tohoku University, Japan

** Faculty of Education, Tokoha University, Japan
}

Information and Communication Technology (ICT) has resulted in reduced lesson preparation time ${ }^{[3]}$. "Grade processing" includes tasks, such as the processing of grades, creation of test questions, grading/evaluation, compilation of report cards, creation of school reports, and creation of cumulative guidance student records ${ }^{[1]}$. In connection with the compilation of report cards and creation of school reports and cumulative guidance student records, a study has revealed that teachers "feel that the situation regarding school administrative work has improved" following the introduction of teachers' school work support systems ${ }^{[4]}$. However, few studies have reported a reduction in the burden of scoring tests, which is another major component of grade processing. To address this shortcoming, this study focuses on estimating and reducing the burden of grading on teachers working in primary and secondary education.

Tests administered by primary and secondary school teachers include quizzes, unit tests conducted at the ends of teaching units, periodical tests conducted at the ends of semesters, and grade tests. A survey by the Benesse Educational Research and Development Institute $^{[5]}$ (henceforth referred to as "Benesse") revealed that in junior high schools the results of periodical tests tend to be weighted most heavily during the preparation of report cards, while in primary schools the results of unit tests were accorded the highest weight. The current study thus aims to reduce the burden of 
grading responsibilities pertaining to the two aforementioned types of tests.

While graded tests are sometimes referred to as "examinations" in certain contexts, we refer to them as "tests" in this study.

\subsection{Characteristics of Tests Based on Type of School}

In primary and secondary education in Japan, schools follow the subject-teacher system and the classroomteacher system depending upon their preferences. Junior and senior high schools generally utilize the subjectteacher system. In public primary schools, the system to assign teachers to classes depends on the type of school-while certain primary schools follow the subject-teacher system corresponding to certain grades and subjects, the majority of schools follow the classroomteacher system ${ }^{[6]}$.

Tests administered by subject teachers exhibit different characteristics from those administered by classroom teachers. According to the Systems Research \& Development Institute of Japan ${ }^{[7]}$, the majority of teachers employed in junior and senior high schools following the subject-teacher system teach a single subject, whereas most teachers working in primary schools, which tend to prefer the classroom-teacher system, teach six or more subjects. According to Benesse ${ }^{[8]}$, the majority of teachers working in junior and senior high schools following the subject-teacher system use their own educational materials as the basis of periodical tests, whereas most teachers working in primary schools following the classroom-teacher system use commercially available evaluation tools for unit tests.

Further, according to Benesse ${ }^{[9]}$, periodical tests are administered approximately five times a year in junior and senior high schools following the subject-teacher system. According to interviews conducted by the first author with 12 teachers working in national, public, and private junior and senior high schools following the classroom-teacher system, the number of questions on each test is approximately 60 corresponding to each subject; and the number of test takers is approximately 120 on average over all the classes. In addition, based on the first author's inquiries at a company specialized in developing and selling educational materials as evaluation tools for unit tests to primarily public primary schools, unit tests are conducted 60 times per year across all subjects on average in primary schools utilizing commercially available unit tests. Further, the number of questions per test for each subject is approximately 25. A similar trend was observed in connection with other companies supplying educational materials as well. The average number of test takers in a single class is approximately 30 . Test characteristics, sorted by the type of school, are summarized in Table 1.

As is evident from the table, in junior and senior high schools following the subject-teacher system, teacher-created tests are predominantly used, and teachers are required to make a large number of correct/incorrect judgments corresponding to each round of scoring. In contrast, in primary schools following the classroomteacher system, commercially available unit tests are usually utilized, and each teacher is responsible for handling a significant number of tests and subjects.

\subsection{Test Workflow and Classification/ Definition of Scoring Procedures}

In this study, test-related tasks conducted by teachers were divided into three processes - preparation, implementation, and scoring (Fig. 1) - based on materials provided by Japan Association for Research on Testing $^{[10]}$ and the Study Group on the Use of School

Table 1. Major characteristics of tests in each type of school

\begin{tabular}{|c|c|c|c|c|c|c|}
\hline Type of school & $\begin{array}{c}\text { Scorer } \\
\text { (Subject scored) }\end{array}$ & $\begin{array}{l}\text { Tests linked to } \\
\text { grade processing }\end{array}$ & Tests used & Tests/year & Questions/test & $\begin{array}{l}\text { Test takers/scorer } \\
\text { (classes tested) }\end{array}$ \\
\hline $\begin{array}{c}\text { Junior/senior } \\
\text { high school }\end{array}$ & $\begin{array}{l}\text { Subject teacher } \\
\text { (Single subjects) }\end{array}$ & Periodical tests & $\begin{array}{c}\text { Teacher-created } \\
\text { tools }\end{array}$ & Approx. 5 & Approx. 60 & $\begin{array}{c}\text { Approx. } 120 \\
\text { (multiple classes) }\end{array}$ \\
\hline $\begin{array}{l}\text { Primary } \\
\text { school }\end{array}$ & $\begin{array}{l}\text { Classroom teacher } \\
\text { (multiple subjects) }\end{array}$ & Unit tests & $\begin{array}{l}\text { Commercially } \\
\text { available tools }\end{array}$ & Approx. 60 & Approx. 25 & $\begin{array}{l}\text { Approx. } 30 \\
(1 \text { class })\end{array}$ \\
\hline
\end{tabular}

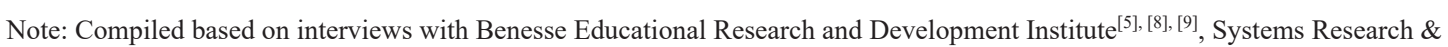
Development Institute of Japan ${ }^{[10]}$, and Kyoikudojinsha Co., Ltd. 
Educational Materials ${ }^{[11]}$. The preparation of commercially available unit tests is significantly different from that of teacher-created tests-questions are "selected" in the former case rather than "created," and tests are "purchased" rather than "duplicated" for the number of test takers. However, the procedures for implementing and scoring tests remain identical in both cases.

The various scoring procedures examined in the study can be defined as follows.

(1) Judgment of Correctness of Answers: The procedure to determine whether the answers provided by test takers are correct or incorrect.

(2) Score Calculation: The procedure to calculate the test takers' scores and compiling it on the answer sheets following correct/incorrect judgments.

(3) Score Recording: The procedure to transfer (enter) the test takers' scores into the recording sheet or teachers' school work support system.

\subsection{Prior Research}

Various systems have been proposed over the years to reduce the burden of test scoring among teachers. Usually, they employ computer programs to perform correct/incorrect judgments of answers and automate the score calculation and score recording processes. The most typical of these is the marksheet method, which is often employed in large-scale tests. Its preparation procedure requires teachers to create selective question and answer columns on the question and answer sheets specifically for the mark-sheet method. Therefore, teachers who administer writing tests are additionally burdened by the test-creation process during the implementation of the mark-sheet method. Moreover, none of the commercially available unit tests used in primary schools employ the mark-sheet method.

The computer-based testing (CBT) method, in which the test itself is administered by a computer, offers a variety of advantages. Besides automating the scoring process via computerization, it also promises to let learners choose their preferred test days based on item response theory (IRT) in the foreseeable future ${ }^{[12]}$. The implementation of this method involves the creation of question and answer forms by teachers for the benefit of the CBT system. It also requires preparation computers to be used by learners. However, according to "Results of the 2016 Survey on the State of Computerization of School Education"[13], as of March 1,2017 , the average number of students per educational computer in Japanese public primary and secondary schools is 5.9. It is, therefore, difficult to implement CBTs based on the current level of ICT provisions.

Another automatic scoring method requires tests to be conducted and scored using special paper and pen devices (henceforth referred to as the "P\&PD method") ${ }^{[13]}$. While the P\&PD method allows the use of existing tests, it requires the creation of specialized answer sheets based on special paper imprinted with a dotted pattern and the use of special pens by test takers to record their answers on the paper. In other words, the P\&PD method is also logistically problematic due to the additional work that must be conducted by teachers during the preparation and implementation of tests.

Extensive research has also been conducted to reduce the burden of grading among teachers without altering existing procedures or introducing additional procedures during the test preparation and implementation stages. In one of the proposed methods, answer sheets for writing tests are inputted into a computer after converting them into digital images using a scanner equipped with an automatic document feeder (ADF). Subsequently, identical answer fields of students taking the same test are processed as images and displayed as skewered stacks (henceforth referred to as the "imageskewered method"). To implement the image-skewered method, teachers are required to configure settings and

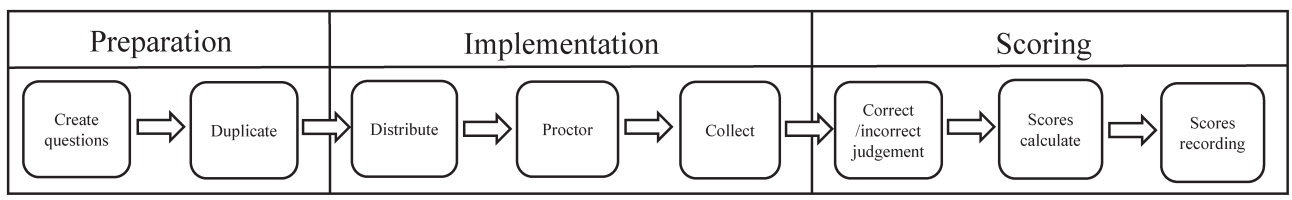

Figure 1. Test procedure

Note: Compiled with reference to Japan Association for Research on Testing ${ }^{[10]}$ and Study Group on the Use of School Educational Materials $^{[11]}$. 
coordinate positions of the answer fields and areas (henceforth referred to as "area settings") before correct/incorrect judgements. This method is particularly advantageous as it does not introduce any additional procedures, such as arrangement of special papers or devices, during the preparation and administration of tests, and existing writing tests can be used without any alteration.

"Development of Marking-support Soft in Taskgiven-type Examination" is a study investigating system development based on the image-skewered method ${ }^{[15]}$. The study involved an experiment and a follow-up questionnaire, in which the amounts of time consumed to score tests were calculated and compared with that consumed during manual scoring using a system developed by the authors (henceforth referred to as the "Takeuchi system"). 16 university students who participated in the experiment were used as scorers, and they used a section of responses on a university test. The analysis of the experimental results revealed that "software-based marking is faster and generates fewer mistakes." In addition, the results of the questionnaire indicated that "many users considered software-based marking easier and more accurate."

A closer look at the experimental data recorded by Takeuchi and Sakuma ${ }^{[15]}$ revealed a $16.0 \%$ reduction in marking duration by using automatic scoring method, expressed by ([Time taken for manual scoring] - [Time taken for scoring using the Takeuchi method]) / [Time taken for manual scoring]. However, results of individual experiments demonstrated that 4 of the 16 participants $(25.0 \%)$ completed manual scoring more quickly compared to the Takeuchi system.

The reported experimental data did not include the time required to input the answer sheets into the scanner. To account for this duration, the scoring period required by the Takeuchi system was recalculated by including the time required to scan the answer sheets (74 seconds for 15 answer sheets), as recorded by Takeuchi and Sakuma ${ }^{[15]}$. This resulted in a $4.1 \%$ reduction in scoring duration compared to manual scoring. The results also demonstrated that 6 of the 16 participants $(37.5 \%)$ completed manual scoring in less time, suggesting significant room for improvement in the performance of the Takeuchi system. Further, Takeuchi and Sakuma $^{[15]}$ did not conduct experiments in primary or secondary schools and did they comment on the comparison between scoring durations required by subject teachers scoring multiple classes of students and classroom teachers scoring different types of commercially available unit tests.

The Takeuchi system is operationally convenient as it can be installed and utilized simply by copying the system files used in a single operation. However, its development has been already terminated, thereby providing no operation guarantee to the currently supported client operating systems.

In addition, a server-client system based on the image-skewered method was developed by Liu and Aoki ${ }^{[16]}$ to support the scoring of large-scale tests involving more than 2,000 test takers. A number of commercial products are also available (Digiraku Scoring 2 by SCANET Inc., ${ }^{[17]}$ and Saiten no Tatsujin by Primagest, Inc., ${ }^{[18]}$ etc.). However, such systems are designed for tests in the context of large university lectures or large-scale tests such as entrance and mock examinations. As a result, they do not take into account the test characteristics of the various types of primary and secondary schools depicted in Table 1. Therefore, systems developed in the aforementioned studies are illsuited for application in primary and secondary education.

\section{Purpose and Method of Study}

\subsection{Purpose}

The purpose of this study is to develop and evaluate a scoring support system to reduce the burden of scoring among teachers of primary and secondary schools (henceforth referred to as "the system") with reference to existing research and aforementioned test characteristics determined by the types of schools by considering the two following perspectives.

Perspective (1): Does the system reduce the required scoring duration corresponding to infrequently conducted tests involving large numbers of questions and test takers (over multiple classes), as is the case for periodical tests conducted by subject teachers, compared to manual scoring?

Perspective (2): Does the system reduce the required scoring duration in the case of frequently conducted tests involving small numbers of questions and test takers, as is the case for unit tests conducted by classroom teachers, compared to manual scoring? 


\subsection{Methodology}

The study was conducted by implementing the following procedure.

(1) Consider and design the necessary functions for a scoring support system to reduce the burden of scoring responsibilities among subject and classroom teachers in primary and secondary schools.

(2) Develop the system designed in (1).

(3) Utilize manual scoring and system-based scoring to execute scoring in tests conducted by subject and classroom teachers, and compare the durations required by the two methods.

\section{System Design}

The system developed in this study was subject to the following requirements.

Requirement (1): The creation of new tests to fit the system should not be required, and existing writing tests should be implementable within the system.

Requirement (2): Teachers should be able to start using the system based on simple operations without having to create a large-scale system environment.

Requirement (3): The time required for scoring by the system should be shorter than that required by the conventional manual scoring method.

Requirement (4): The system should exhibit the following functions to address the test characteristics of each type of school.

A. It should exhibit a function to handle various types of commercially available unit tests used in the classroom-teacher system.

B. It should exhibit a function to score tests corresponding to multiple classes under the subjectteacher system.

\section{System Development}

To satisfy requirement (1) of the system design, the image-skewered method developed in a previous study was employed, and an associated scoring support system was developed with functions enabling teachers to use existing writing tests without alterations, as in case of the previously discussed Takeuchi system. The scoring results were stored in a database within the system and outputted as .csv files. Images of the scored answer sheets were generated in the system and outputted using a printer.

\subsection{Operating Environment}

The system is taken to be a standalone application developed in Visual C\# using an SQLite database, which runs on .NET Framework in Windows 7 or any later operating system (32-bit and 64-bit). The necessary hardware was established to require at least $4 \mathrm{~GB}$ of memory, $100 \mathrm{MB}$ of available hard-disk space, and a screen resolution equal to or greater than $1024 \times 768$ pixels.

To satisfy requirement (2), the system was endowed with a function allowing the application to be operated by copying its system files, as in the case of the Takeuchi system.

\subsection{System Functions}

\subsubsection{Function Operate Only at Incorrect Locations}

The Takeuchi system also employed the image-skewered method, and when making correct/incorrect judgments "only images of the answer sections are extracted, and then allocated to the score folders manually by the scorer" ${ }^{2[15]}$. Therefore, it was necessary to perform an operation on all answers, irrespective of whether they are correct or incorrect. The other systems proposed in existing studies also required all answers to be assigned with a correct or incorrect mark, or to be marked as partially correct.

To satisfy requirement (3), the system was endowed with a function which assigns an incorrect mark whenever the location of an incorrect answer is clicked once during the assessment stage, and assigns a partially correct mark whenever the relevant location is clicked twice. The system was also equipped with a new additional function which registers all unclicked answer locations as correct answers in the system (henceforth referred to as the "function operating only at incorrect locations"; see Fig. 2). This function eliminates the need to evaluate all answer locations and is, therefore, expected to reduce the time required to make correct/ incorrect judgements.

\subsubsection{Area Setting Package Function}

In the Takeuchi system, the area setting procedure conducted prior to correct/incorrect judgements required the user to "designate the answer extraction area" ${ }^{[15]}$. 
Similarly, the system developed in this study requires teachers to configure the areas of the answer fields. However, the need to individually define the relevant areas corresponding to each test creates an additional burden for classroom teachers, who conduct around 60 unit tests per year using commercially available tests, as presented in Table 1 . Thus, to satisfy requirement (4)A, the system was endowed with a new function to save the initial area setting information as an XML file, enabling the settings to be reapplied as an area setting package (henceforth referred to as the "area setting package function"; consult Fig. 3).

This function is also expected to reduce the time required for scoring, as it enables teachers to conduct correct/incorrect judgements without having to redefine the areas before each test by configuring an area setting package for the tests in advance. In addition to classroom teachers who use commercially available tests, the

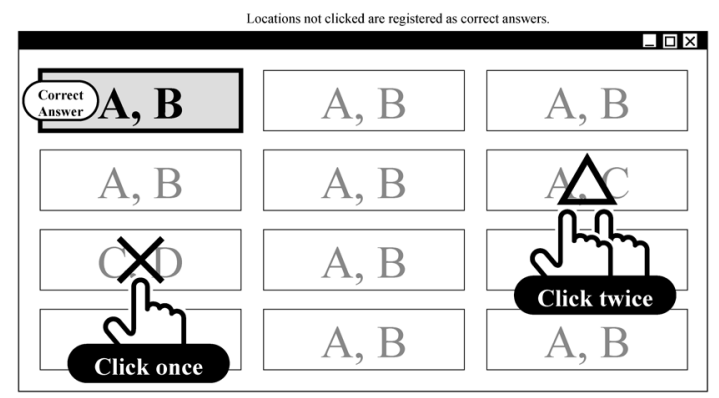

function enables subject teachers to use the same tests for multiple classes.

\subsubsection{Multiple Class Selection Function}

As depicted in Table 1, subject teachers administer and score the same tests for multiple classes. However, in the Takeuchi system and the other systems proposed in existing studies, no functions are used to simultaneously score multiple classes taking the same test in a single batch. This required teachers to prepare student lists containing the names of the test takers corresponding to each instance of scoring. Therefore, to satisfy requirement (4)B, the proposed system was endowed with a new function to select multiple classes taking the same test and simultaneously score them in a single batch, by preparing a student list only once upon class organization (henceforth referred to as the "multiple class selection function"; consult Fig. 4). The function can be used by entering the details of each homeroom or tracked class into the student list imported into the system.

Because the multiple class selection function eliminates the requirement of recompilation and import of student lists corresponding to each instance of scoring, the system developed in this study is expected to afford greater convenience of usage than existing methods.

Figure 2. Function to operate only in incorrect places

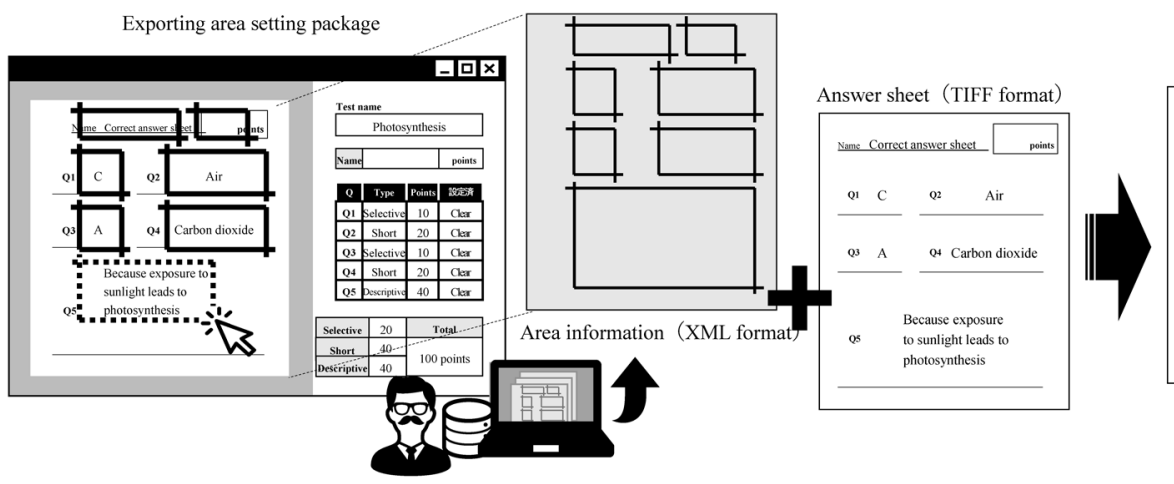

Importing area setting package

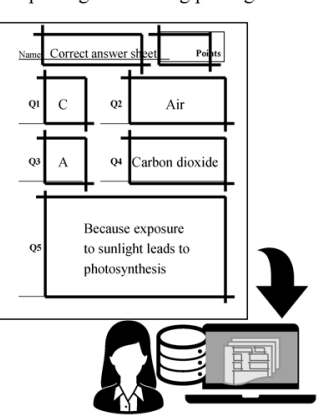

Figure 3. Area setting package 


\section{System Operation Procedure}

To provide a broad overview of the system, an outline of the operation procedure is described below and illustrated in Fig. 5.

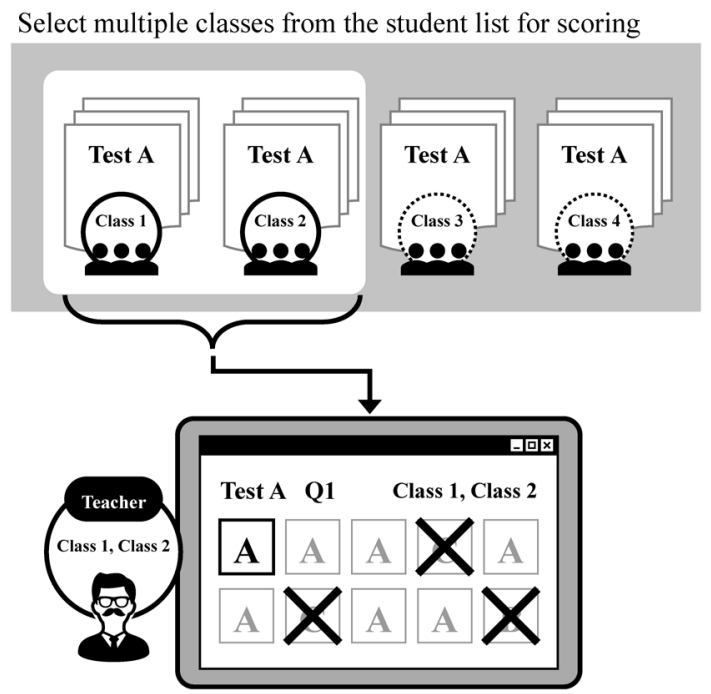

\subsection{Importing the Student List}

Scoring via the system requires the student list to be imported into the system. The student list is entered into an Excel file with fields corresponding to student ID, name, grade, class, and student number within the class. Teachers can also enter information on tracked classes, whenever necessary. This procedure is required to be performed only once upon class organization, and does not need to be repeated corresponding to each scoring instance.

\subsection{Scanning Correct Answers and Answer Sheets}

The correct answers and answer sheets are scanned and imported into the system in the form of TIFF image files (Fig. 5a). If the writing on the answer paper is not adequately prominent, the relevant concentration can be adjusted using the scanner settings.

Figure 4. Multiple class selection function

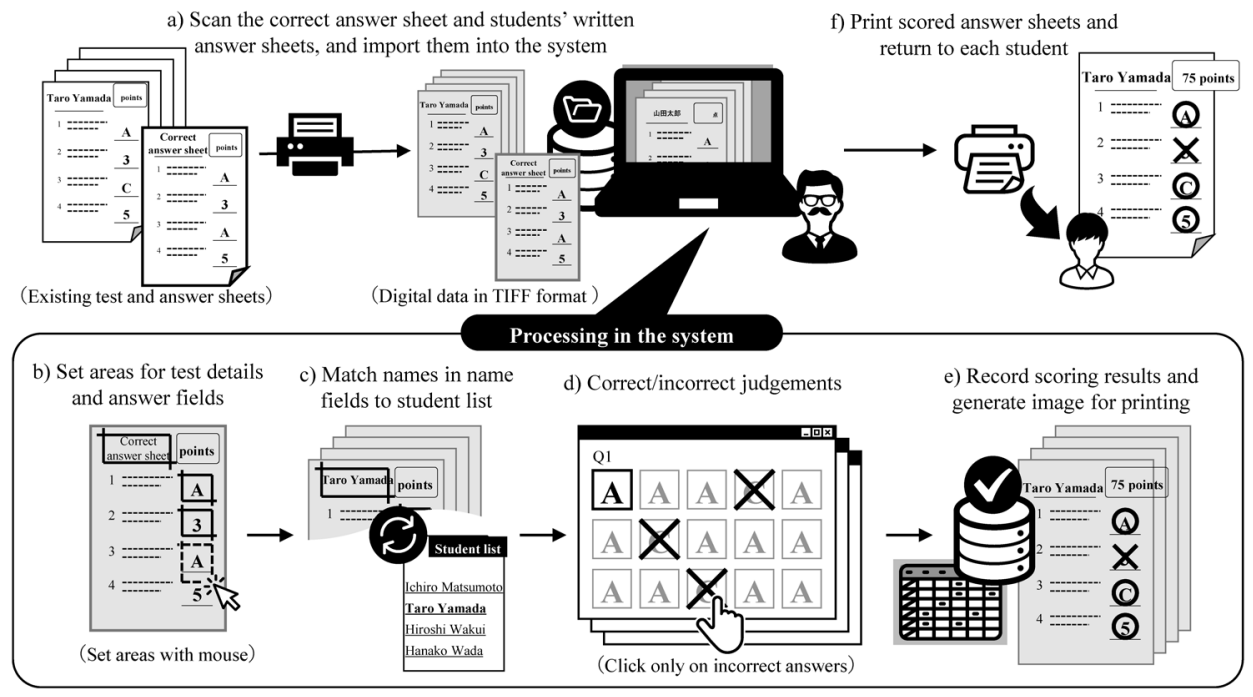

Figure 5. System operation procedure 


\subsection{Configuring the Test Details and Areas}

The subject, grade, test type, and paper size were configured based on the image files of the correct answers imported into the system, and the areas containing the student's name, total score, and answer columns were defined using the mouse. Area settings were configured to allocate the location corresponding to each answer field (Fig. 5b). In case of previously configured area setting information, the teacher could import this information by utilizing the area setting package function, thereby eliminating the need to reconfigure the settings corresponding to each scoring instance (Fig. 3).

\subsection{Name Matching}

The names in the student list imported into the system were matched with the student names appearing in the answer sheet name fields (henceforth referred to as "name matching"; consult Fig. 5c). During the scoring of answers of students from multiple classes taught by a single subject teacher, the name matching procedure was conducted for the relevant classes using the multiple class selection function (Fig. 4).

\subsection{Correct/Incorrect Judgement}

Corresponding to each correct/incorrect judgement, the answers provided by all test takers were displayed on a single screen. The scorer performed judgment by clicking on the locations of incorrect answers (Fig. 5d). Using the function operating only at incorrect locations (consult Fig. 2), answers not clicked by the scorer were registered as correct answers within the system database, and all clicked answers were registered as incorrect.

\subsection{Recording and Output of Scoring Results}

After the completion of correct/incorrect judgements, the results of all test takers corresponding to each question were displayed in a list. Following the judgement phase, the system automatically compiled information on score calculations and score recording and recorded it in the system database (Fig. 5e). Scored answer sheets containing marks denoting correct and incorrect answers and total scores were then printed and returned to the test takers (Fig. 5f). In addition, the score results were outputted as a .csv file using the score recording function. The system was also designed to be capable of recording the durations required by each procedure in the scoring process.

\subsection{Comparison Between Scoring Procedures}

As discussed previously, the procedure for system-based scoring is different from that of manual scoring (Fig. 6). First, correct/incorrect judgements were performed after scanning the correct answer sheets into a computer, configuring the areas based on the correct answer sheet, and matching the names on the answer sheets to the names appearing in the student list imported into the system in advance. The score calculation and score recording procedures, usually conducted via manual scoring, were handled automatically by the system, thus eliminating the need for the scorer to repeatedly perform these steps. Further, the names only needed to be imported once during class organization, rather than corresponding to every scoring instance.

\section{Evaluation}

Experiment 1 was conducted to determine the reduction in scoring duration by using the system in schools following the subject-teacher system, in consonance with Perspective 1. Experiment 2 was conducted to determine the reduction in scoring durations by using the system in schools following the classroom-teacher system, in consonance with Perspective 2. The two experiments are described below in detail.

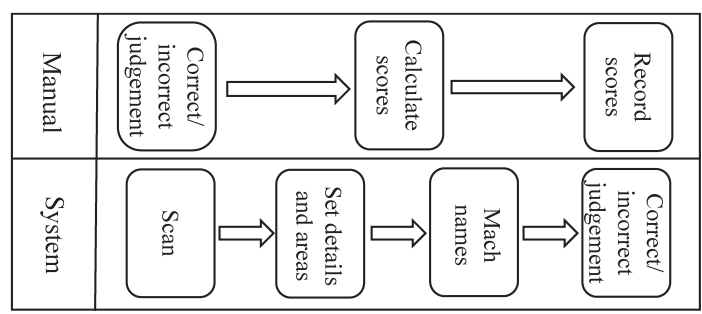

Figure 6. Comparison of scoring procedures 


\subsection{Experiment 1: Scoring by Subject Teachers}

\subsubsection{Overview}

The experiment was designed to determine the reduction in scoring duration achieved by the implementation of the system with respect to teacher-created periodical tests administered by subject teachers. The durations required to complete each scoring procedure via systembased scoring were measured and compared with those achieved via manual scoring corresponding to teachercreated periodical tests administered by subject teachers during class. The first author also observed the teachers performing the system-based scoring procedure and some of the manual scoring procedures.

\subsubsection{Target Tests and Schools}

The experiment focused on periodical tests administrated for various grades and subjects in one private primary school, two private junior high schools, one private senior high school, and one public senior high school, each of which followed the subject-teacher system. 12 separate tests were conducted at the target schools between late November, 2017, and late May, 2018. The tests were scored by the subject teachers who taught the respective classes and administered the tests. Details of the tests for which the scoring durations were measured are presented in Table 2.

Although scoring was conducted by ten teachers, there were 12 scoring IDs, since IDs 1, 2, and 7 were assigned to tests scored by the same teacher. Ten different tests were conducted, with IDs 9,10 , and 11 being assigned to the same test taken by students in separate tracked classes from the same school/grade. In addition, IDs 9,10 , and 11 were assigned to tests scored by three different teachers in charge of the separate tracked classes.

The average number of test takers corresponding to each scorer was 73.5; the average number of questions was 60.5 , of which an average of 30.8 were selective questions and the rest were descriptive questions. The total number of answer locations was 4,721 on average. In addition, the answer formats for questions used in all tests examined in this study were classified with reference to the National Institute for Educational Policy Research $^{[19]}$. The computers used by teachers who participated in the experiment to access the system ran on an operating environment that supported installation of the system. Finally, each school was equipped with scanners with automatic ADF units.

\subsubsection{Methods to Measure Scoring Durations}

Corresponding to each scoring ID, the durations required by manual scoring and system-based scoring were calculated and recorded using the following methods. The scoring periods and respective scoring durations are presented in Table 2.

Except the cases of scoring IDs 2 and 7, systembased scoring was conducted after the completion of manual scoring. As the scoring durations required by system-based scoring was likely to be influenced by the memory and experience of the scorer after completing the manual scoring, an intervening period ranging between several days and several weeks was allowed to pass between the performances of the two forms of scoring. However, in the case of scoring IDs 1, 2, and 7, manual scoring and system-based scoring were conducted on the same day because it was not possible to secure separate times for system-based scoring owing to constraints concerning the scorers' school affairs.

In addition, the methods used by each scorer when performing correct/incorrect judgements during manual scoring were defined beforehand. Correct/incorrect judgements for a single question for all test taker at the same time were referred to as the "problem-by-problem correct/incorrect judgement method" (henceforth referred to as the "P method"). Further, all assessments corresponding to each test taker was referred to as the "each answerer correct/incorrect judgement method" (henceforth referred to as the "S method"). The tests were divided into blocks corresponding to each perspective or question-section based on the "question blockby-block correct/incorrect judgement method" (henceforth referred to as the "B method").

During the calculation of the time required for manual scoring, the scorers separately measured the times required for correct/incorrect judgements, score calculation, and score recording using a watch or a stopwatch. As scorers with scoring IDs 7 and 8 performed the score calculation and score recording steps within a single process, the total time for both procedures was recorded as the score recording duration in their cases. Following the measurements, the durations were recorded in minutes. 


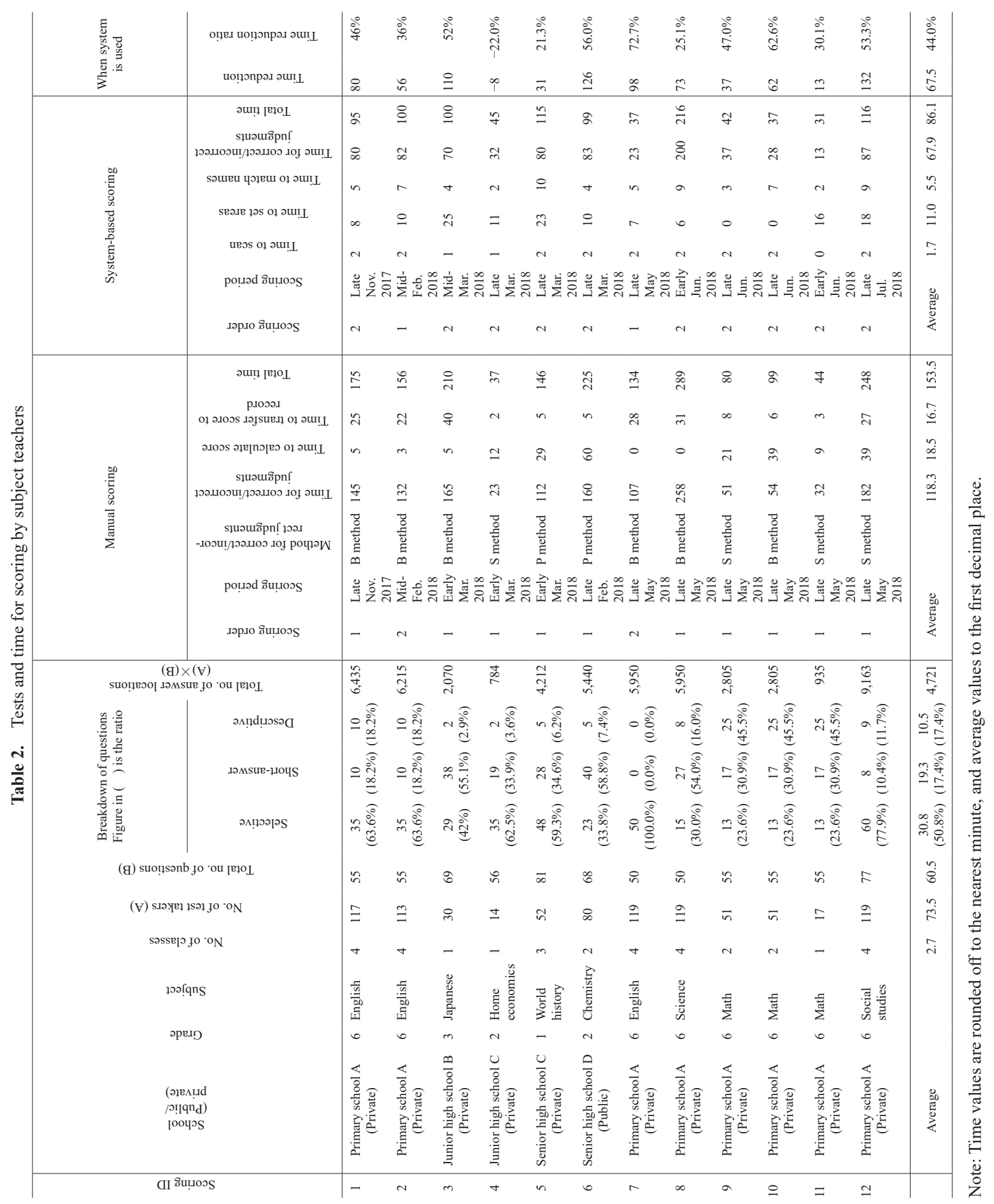




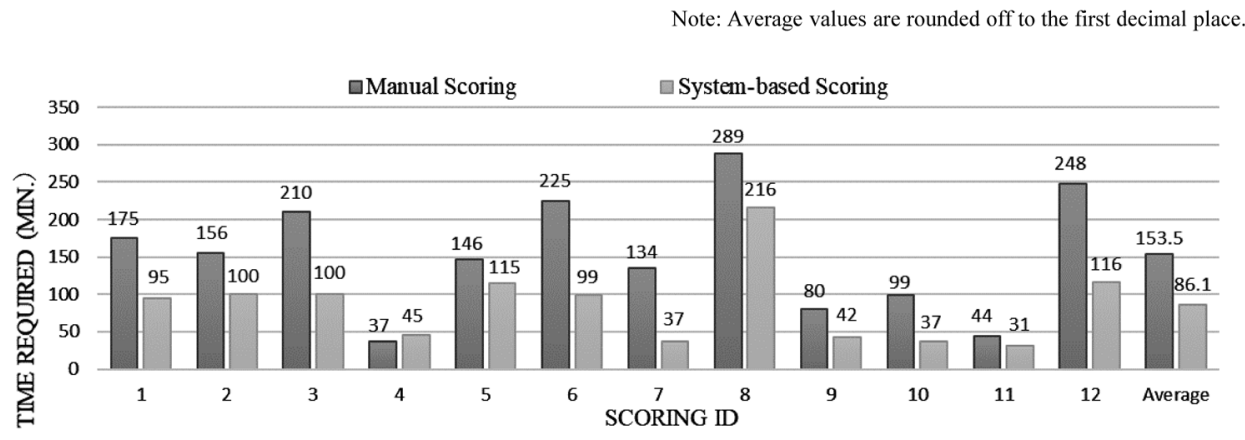

Figure 7. Comparison of scoring times for subject teachers

During the calculation of the durations required by system-based scoring, the durations required for scanning, area setting, name matching, and correct/incorrect judgements were separately measured. The durations required to scan the answer sheets were measured by either the first author or the scorers, while the other durations were obtained from the system operation logs. Following the completion of measurements, the times were recorded in minutes to correspond to the method used during manual scoring. In addition, as scoring IDs 9 and 10 corresponding to the tracked classes were based on the same test as scoring ID 11, the area setting package configured for scoring ID 11 was reapplied, resulting in an area setting time of $0 \mathrm{~min}$ in their cases. The scoring dates and the methods of manual scoring used by the scorers are presented in Table 2, along with the corresponding scoring durations.

The scorers were observed to be able to easily use the system after the first author installed it on their computers and provided instructions on its use. After initialization, the scorers imported their prepared student lists into the system. Although this procedure required approximately 20 minutes in total, it was only required to be performed once. Hence, it was not included in the total time required by system-based scoring.

\subsubsection{Results}

Manual scoring and system-based scoring durations were compared corresponding to the 12 aforementioned tests conducted by 10 subject teachers (Fig. 7). The average duration required by manual scoring was observed to be 153.5 minutes, while that required by system-based scoring was only 86.1 minutes. Therefore, in this experiment, system-based scoring exhibited a reduction in average duration of approximately 67.5 minutes $(44.0 \%)$ compared to manual scoring (Table 2). Further, the duration required by system-based scoring was shorter than that required by manual scoring in 11 of the 12 cases, except the case of scoring ID 4 .

\subsection{Experiment 2: Scoring by Classroom Teachers}

\subsubsection{Overview}

This experiment was performed to determine the reduction in scoring duration achieved by the implementation of the system in the context of commercially available unit tests administered by classroom teachers. The durations required to complete each scoring procedure were recorded and compared between manual scoring and system-based scoring. The system's area setting package function was used during system-based scoring. In this case as well, the first author observed the teachers performing system-based scoring and some of the manual scoring procedures.

\subsubsection{Target Tests and Schools}

The experiment focused on scoring tests in various subjects taught between the second and sixth grades in two public primary schools following the classroom-teacher system. The tests were conducted a total of eight times at the target schools, between late April, 2018, and late May, 2018. The tests were scored by the classroom teachers who taught the respective classes and administered the tests. The tests for which the scoring durations were measured are summarized in Table 3. 
Scoring was conducted by three teachers and scoring ID pairs 13 and 16, and 18 and 20, were assigned to tests completed by the same teachers. The eight tests were of different types.

The average number of test takers for each scorer was 31; the average number of questions was 24.1 , of which an average of 9.6 were selective questions, 12.5 were short-answer questions, and 1.9 were descriptive questions. The total number of answer locations was 743.5 on average. Since considerable paperwork was required to install the software on the computers used for school-work by the teachers who participated in the experiment, a computer was prepared in advance by the first author to be used with the permission of the school principal. In addition, all personal information was deleted from the computer after the experiment. Corresponding to scoring IDs 13 to 16 , the school's $\mathrm{ADF}$ unit-equipped scanner was used; and for IDs 17 to 20 , a scanner prepared by the first author was used with the permission of the school principal.

\subsubsection{Scoring Time Measurement Methods}

Corresponding to each scoring ID, the durations required by manual scoring and system-based scoring were calculated and recorded using the following methods. The scoring periods and respective durations are presented in Table 3.

Except the case of ID 16, system-based scoring was conducted following the completion of manual scoring. As the scoring duration required by systembased scoring was likely to be influenced by the memory and experience of the scorer having completed the manual scoring process, intervening periods ranging from several days to several weeks were instituted between the two scoring tests. In addition, the method used by each scorer to perform correct/incorrect judgements during manual scoring was clearly defined beforehand.

The durations required by manual scoring were measured using the same methods described in the previous experiment. Following the completion of measurements, the durations were recorded in minutes. As the scorer with ID 17 performed the score calculation and score recording steps as part of a single process, the total duration required by both procedures was recorded as the score recording duration in this case.

The durations required by system-based scoring were also measured using the methods describe in the previous experiment. Following the completion of measurements, the durations were recorded in minutes to correspond to the method used for manual scoring. All unit tests used in this experiment were evaluation tools provided by a company specializing in educational materials, and correct answer sheets were provided along with the questions in advance. Based on these documents, the first author configured an area setting package for the unit tests to be used during systembased evaluation and provided it to the scorers. As a result, the duration required by the scorers to set the area was $0 \mathrm{~min}$ in all cases.

\subsubsection{Results}

The durations required by manual scoring and systembased scoring were compared corresponding to eight tests conducted by three classroom teachers (Fig. 8). The average duration required by manual scoring was observed to be 25.2 minutes, while that required by system-based scoring was only 14.4 minutes. Therefore, in this experiment, system-based scoring reduced the scoring duration by approximately 10.8 minutes $(42.9 \%)$ on average compared to manual scoring (Table 3). Further, the durations recorded by system-based scoring were shorter than those required by manual scoring in all eight cases.

\section{Discussion}

The results of both Experiment 1, with subject teachers, and Experiment 2, with classroom teachers, demonstrated that the average scoring durations required by system-based scoring were shorter than those required by manual scoring in almost all cases.

The primary factor behind the reduction in required duration is the reduced time required for correct/incorrect judgements by system-based scoring. The proportion of the total scoring duration spent for correct/incorrect judgements during manual scoring was $77.1 \%$ for subject teachers (calculated from Table 2) and $68.4 \%$ for classroom teachers (calculated from Table 3). Evidently, this was the most time-consuming stage of the scoring process. During the first author's observations of teachers performing this step during manual scoring, the scorers were observed to spend a considerable amount of time recording correct or incorrect marks corresponding to the 


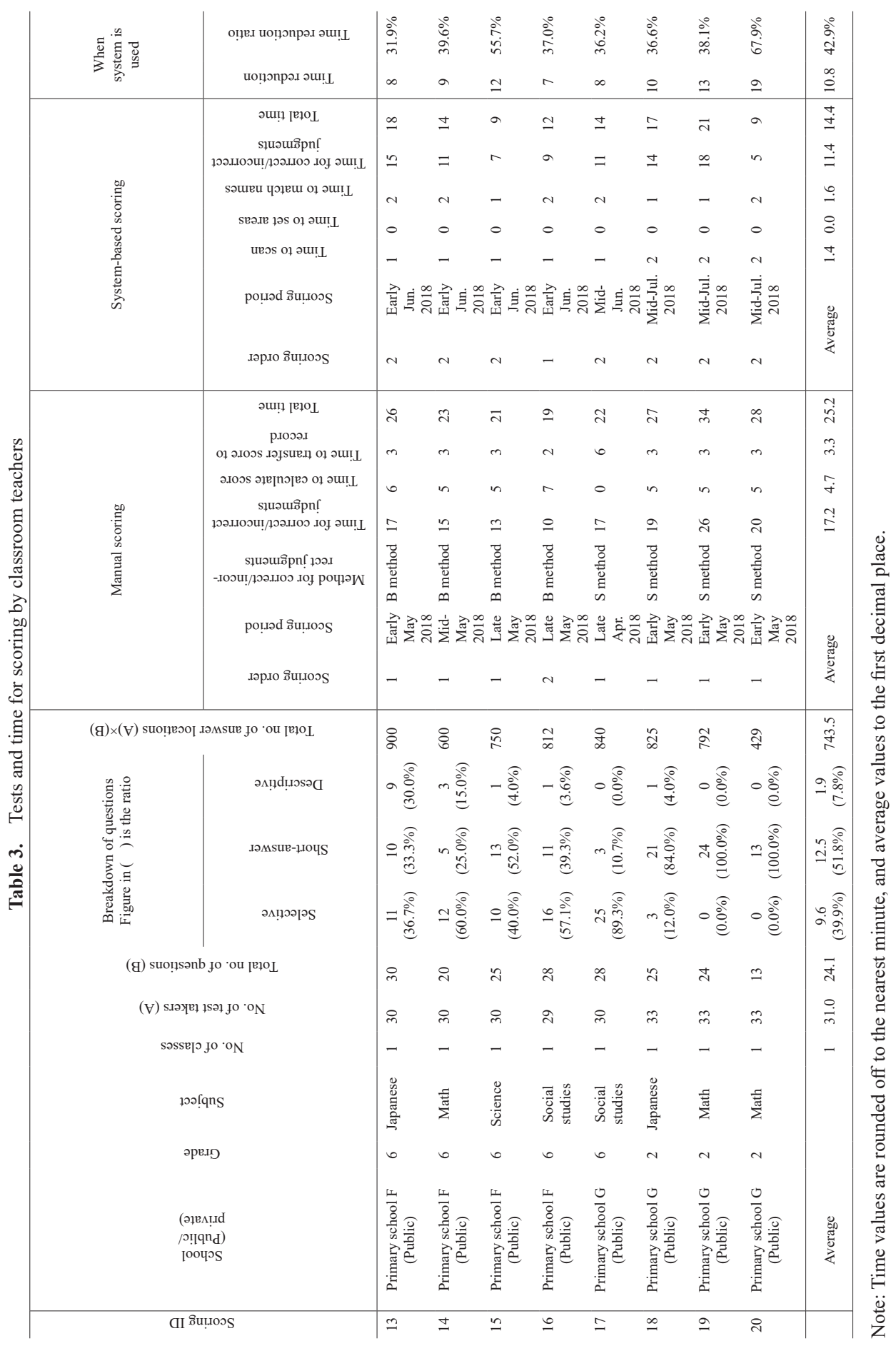




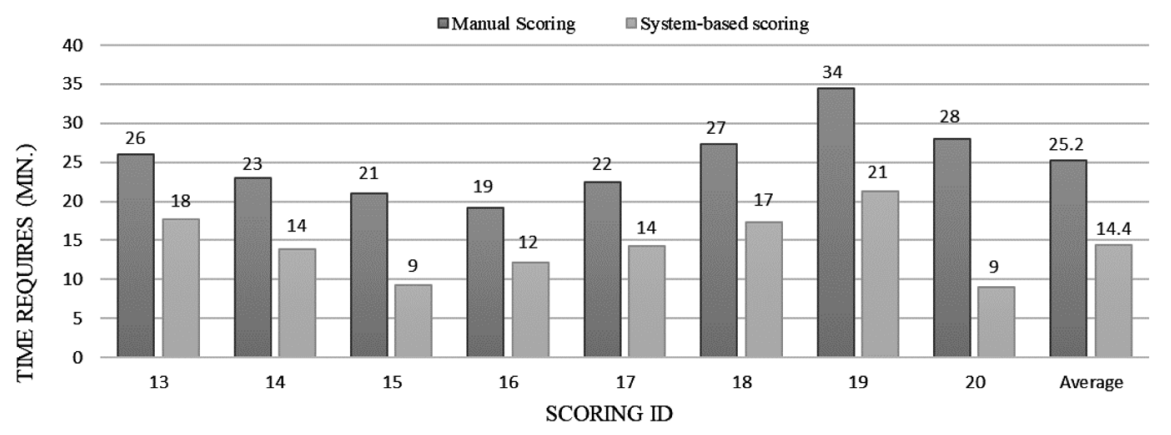

Figure 8. Comparison of scoring times for classroom teachers

answers of all test takers. In contrast, when the system was used to score the tests, scorers used the function operating only at incorrect locations, achieving a time reduction ratio of $42.6 \%$ in Experiment 1 (calculated from Table 2) and $33.8 \%$ in Experiment 2 (calculated from Table 3). As a result, the time spent on correct/incorrect judgements was considerably shorter in the case of system-based scoring compared to manual scoring, leading to a reduction in the total scoring duration.

In addition, the function operating only at incorrect locations shortened the time required for correct/incorrect judgements compared to the Takeuchi system, which also uses the image-skewered method but requires scorers to operate on all answers. According to Takeuchi and Sakuma ${ }^{[15]}$, the Takeuchi system induced a $15.2 \%$ reduction ratio during correct/incorrect judgements, expressed by ([Time taken for manual scoring] [Time taken for scoring using the Takeuchi system])/ [Time taken for manual scoring]. The time reduction ratios for correct/incorrect judgements in both experiments conducted with the system $(42.6 \%$ for subject teachers, $33.8 \%$ for classroom teachers) were significantly greater than those achieved by using the Takeuchi system, demonstrating that the function operating only at Incorrect Locations is effective in reducing the scoring durations.

Finally, we examine the area setting package function, which is another major component of the proposed system. The area setting packages were used during system-based scoring in the experiment with classroom teachers. Therefore, to verify the effectiveness of the area setting package function in reducing the scoring duration, trial calculations were conducted corresponding to hypothetical cases without using setting packages. During the scoring of Experiment 1, the average number of questions per test was 60.5 , and the average time taken to define the areas was approximately 11 minutes (Table 2). Therefore, it is reasonable to assume an average area setting time of 0.18 minutes per question. As the average number of questions per test for classroom teachers was 24.1 (Table 3 ), if the area-setting time per unit test is assumed to be 4.3 minutes, the average time reduction ratio achieved by system-based scoring is $25.8 \%$ (an average time reduction of $6.5 \mathrm{~min}$ ). This also suggests that when the proposed system is used for scoring by classroom teachers, the area setting package function plays an important role in the reduction of the total scoring time.

Based on these results, we can conclude that the system helps to significantly reduce the burden of scoring responsibilities among teachers in various types of primary and secondary schools.

The experimental results demonstrated that manual scoring performed better by eight minutes in only 1 out of the 20 cases considered in aggregate (scoring ID 4 in Experiment 1) compared to system-based scoring. This is because, in the case of scoring ID 4, the time required by system-based scoring during assessment was approximately nine minutes longer than that required by manual scoring. Observation of the scoring procedure in progress indicated that this step took more time due to the difficulty of detecting the scanned images as the teacher with scoring ID 4 had not adjusted the density settings during the scanning of the answer papers. This 
was exacerbated by the fact that the test takers had not written their answers prominently on the papers in this case. This indicates that adequate scanner operating skills are necessary to use the system properly.

\section{Future Research Directions}

The multiple class selection function, which is a major component of the system, enabled subject teachers to select multiple classes after importing the student list only once during class organization. The student list did not need to be imported during each scoring instance, as the multiple class selection function can be used for all 5 to 60 tests per year. This study, which focused on one round of scoring over a limited period, did not measure the durations required to import the student list and use the multiple class selection function itself. Verification of the reduction in duration by implementing the multiple class selection function remains an issue that should be addressed in future research.

Moreover, the calculation of the time reduction ratios corresponding to different question types during the correct/incorrect judgement stage would enable more detailed measurement of time-saving effects of the system. However, one limitation of the present study was that the duration required on each question type in manual scoring was not measured in this study, in view of the burden this would place on individual scorers. Thus, analysis of the time reduction ratios corresponding to correct/incorrect judgements of individual question types remains another issue to be addressed in future research.

Following the completion of Experiment 1, comparison of the scoring durations required by the two methods revealed that some teachers had made erroneous judgements during manual scoring. Other teachers opined that they made fewer mistakes when judging answers using the system compared to using the manual scoring method. Although this point was not addressed completely in this study, these observations indicate the possibility that the system could reduce the number of mistakes made by scorers in correct/incorrect judgements. Thus, in future works, we intend to examine this possibility.

In addition, it appears that the procedure of making correct/incorrect judgements has two aspects - a mechanical aspect during which the test takers' answers are recorded as correct or incorrect, and an educational one during which the learning status of test takers is ascertained based on their answers and then utilized during subsequent teaching. In future works, an analysis of how teachers alter their methods of instructions after using the system to score tests and viewing all answers of all test takers in list form should be conducted.

After scoring using the system, the scored answer sheets were printed out and returned to the respective test takers. Commercially available unit tests used in primary schools are of particular note in this context, as they exhibit integrated question and answer sheets with photos and diagrams printed in color. This may be problematic because of the printing costs incurred to print and return the scored answer sheets. In the future, when learners have access to information terminals, it may be possible to return answer sheets to students as data (henceforth referred to as "digital return"). If digital return becomes feasible, in addition to the reduction of costs, the collation of detailed learning histories, such as whether the student who had received the scored answer sheet reviewed the incorrect answers, may become possible. In the future, it will be useful to examine system development for digital return and the effect of varying policies of returning tests on learners.

Finally, companies specializing in educational materials offering commercially available evaluation tools are expected to begin providing area setting packages so that a greater number of teachers can benefit from the reductions in scoring durations achieved by the area setting package function included in the proposed system.

\section{Acknowledgements \\ We would like to thank all the teachers who participated in interviews and trials related to the development of this system. In addition, we are grateful to Professors Yoko Noborimoto, Kiichiro Okubo, Fumiko Yagisawa, and Shota Itagaki for pro- viding invaluable advice to the study.}

\section{Author notes \\ This paper develops and compiles research presented at Seminar 18-2 of the Japan Society for Educational Technology ${ }^{[20]}$ and the 34th Annual Conference of the Japan Society for Educational Technology ${ }^{[21]}$.}

\section{References}

[1] Ministry of Education, Culture, Sports, Science and 


\section{DEVELOPMENT AND EVALUATION OF SCORING SUPPORT SYSTEM}

Technology, "Gakkō ni okeru Hatarakikata Kaikaku ni kansuru Torikumi no Tettei ni tsuite," (in Japanese), Accessed: Jul. 8, 2020. [Online]. Available: http://www. mext.go.jp/a_menu/shotou/hatarakikata/__icsFiles/afield file/2019/03/18/1414498_1_1.pdf

[2] Ministry of Education, Culture, Sports, Science and Technology, "Kyōin Kinmu Jittai Chōsa (Heisei 28 Nendo) (Kakuteihi) ni tsuite," (in Japanese), Accessed: Jul. 8, 2020. [Online]. Available: https://www. mext.go. jp/b_menu/shingi/chukyo/chukyo3/079/siryo/_icsFiles/ afieldfile/2018/09/28/1409717_4_1.pdf

[3] Katsushika Municipal Honden Elementary School, Kyoshitsu ni ICT ga Yattekita. Tokyo: NTT Publishing (in Japanese), 2014.

[4] A. Miyata, T. Yamamoto, T. Horita, M. Ito, J. Katayama, and H. Suzuki, "Secular change of survey for teachers on school work improvement and needs of functions in use school affairs support system," (in Japanese), Jpn. J. Educ. Technol., vol. 39 (Suppl.), pp. 49-52, 2015.

[5] Benesse Educational Research and Development Institute, "Dai-5-kai Gakushū Shidō Kihon Chōsa (Shōgakkō/ Chūgakkō ban) [2010]," (in Japanese), Accessed: Jul. 8, 2020. [Online]. Available: https://berd.benesse.jp/shotou chutou/research/detaill.php?id=3243

[6] Ministry of Education, Culture, Sports, Science and Technology "Heisei 16-nendo Kōritsu Shō / Chūgakkō ni okeru Kyoiku Katei no Hensei / Jisshi Jōkyō Chōsa no Kekka ni tsuite," (in Japanese), Accessed: Jul. 8, 2020. [Online]. Available: http://www.mext.go.jp/b_menu/shingi/ chukyo/chukyo3/004/siryo/attach/1397182.htm

[7] Systems Research \& Development Institute of Japan, "Gakushū Shidō to Gakushū Hyōka ni taisuru Ishiki Chōsa Hōkoku sho, Report on Survey Contracted from Ministry of Education, Culture, Sports, Science and Technology, 2009," (in Japanese), Accessed: Jul. 8, 2020. [Online]. Available: http://www.mext.go.jp/b_menu/ shingi/chukyo/chukyo3/004/siryo/__icsFiles/afieldfile/ 2010/02/19/1289879_1.pdf

[8] Benesse Educational Research and Development Institute, "Dai-3-kai Gakushū Shidō Kihon Chōsa," (in Japanese), Accessed: Jul. 8, 2020. [Online]. Available: https://berd. benesse.jp/shotouchutou/research/detail1.php?id=3249

[9] Benesse Educational Research and Development Institute, "Dai-5-kai Gakushū Shidō Kihon Chōsa (Kōkō ban) [2010],” (in Japanese), Accessed: Jul. 8, 2020. [Online]. Available: https://berd.benesse.jp/shotouchutou/research/ detail1.php?id=3245
[10] The Japan Association for Research on Testing, Minaosō, Testo wo Sasaeru Kihon no Gijutsu to Kyōiku. Tokyo: Kaneko shobo (in Japanese), 2010.

[11] Gakkō Kyōzai Katsuyō Shidōhou Kenkyūkai, Veteran Sensei Jikiden Work Test no Katsuyōhou. Tokyo: Kyōikudōjinsha (in Japanese), 2014.

[12] E. Muraki, "Computer Based Test (CBT) no Jissi to Riron Kenkyū," (in Japanese), J. Soc. Instrum. Control Eng., vol. 40, no. 8, pp. 549-554, 2001.

[13] Ministry of Education, Culture, Sports, Science and Technology, "Heisei 28-nendo Gakkō ni okeru Kyōiku no Jōhōka no Jittai tou ni kansuru Chōsa Kekka," (in Japanese), Accessed: Jul. 8, 2020. [Online]. Available: http://www.mext.go.jp/a_menu/shotou/zyouhou/detail/139 5145.htm

[14] K. Koyama and M. Nakagawa, "An exam system using pen and paper device," (in Japanese), JPSJ SIG Tech. Rep., vol. 2011-CE-108, no. 8, 2011.

[15] T. Takeuchi and A. Sakuma, "Development of markingsupport soft in task-given-type examination," (in Japanese), J. Jpn. Soc. Inf. Syst. Educ, vol. 21, no. 1, pp. 33-42, 2004.

[16] X. Liu and Y. Aoki, "Kyōtsū Kamoku Kyōiku ni okeru Saiten System Kaihatsu oyobi Jissen," (in Japanese), Comput. Educ., vol. 16, pp. 38-42, 2004.

[17] SCANET Inc., "DigiRaku Scoring 2," (in Japanese), Accessed: Jul. 8, 2020. [Online]. Available: https://www. scanet.jp/wp/?p=3002

[18] Primagest, Inc., "Saiten no Tatsujin," (in Japanese), Accessed: Jul. 8, 2020. [Online]. Available: https://www. primagest.co.jp/solution/sol-saiten.html

[19] National Institute for Educational Policy Research, "Heisei 30-nendo Zenkoku Gakuryoku / Gakushū Jokyō Chōsa Kaisetsu Shiryō, Shōgakkō, Sansū," (in Japanese), Accessed: Jul. 8, 2020. [Online]. Available: http://www. nier.go.jp/18chousa/pdf/18kaisetsu_shou_sansuu.pdf

[20] S. Nakagawa, K. Sato, R. Saito, and T. Horita, "Scoring support system in writing test for reducing teacher's burden in primary and secondary educational institutions," (in Japanese), Jpn. J. Educ. Technol., Compilation Res. Rep., vol. 18, no. 2, pp. 223-230, 2018.

[21] S. Nakagawa, K. Sato, R. Saito, and T. Horita, "Development and effectiveness of scoring support system for writing test: Focusing on reducing teacher's burden in Elementary School," (in Japanese), in 34th Annu. Conf. JSET, vol. 34, pp. 777-778, 2018. 


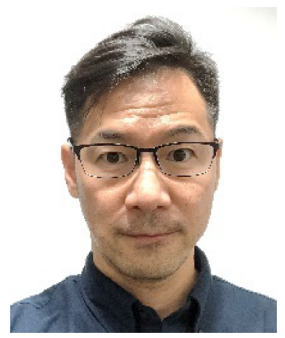

Satoshi Nakagawa is currently a student at the Graduate School of Information Sciences, Tohoku University. He is the CEO of EdLog Co., Ltd. and the Special Inspector of Elementary and Secondary Education Bureau, Ministry of Education, Culture, Sports, Science and Technology Government of Japan. He is a member of JSET and JSISE.

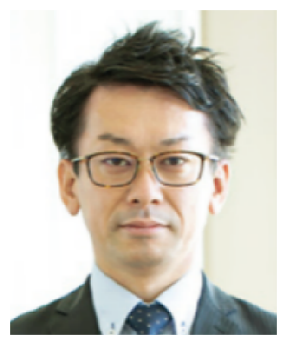

Kazunori Sato received his Ph.D. from the Graduate School of Information Sciences, Tohoku University. He is currently an assistant professor at the Faculty of Education, Shinshu University. His research interests include the development of education in the media and information society. $\mathrm{He}$ is a member of JSET and JAEMS.

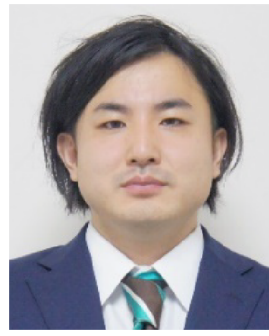

Ryo Saito received his Ph.D. from the Graduate School of Information Sciences, Tohoku University. He is currently an assistant professor at Miyagi University of Education. His research interests include Cognitive Psychology, Educational Psychology, and Cognitive Science. He is a member of JSET.

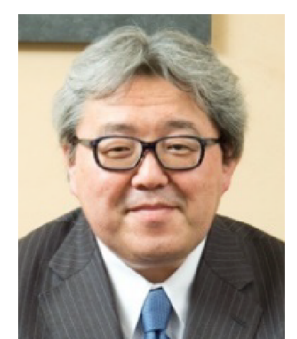

Tatsuya Horita received his Ph.D. from Tokyo Institute of Technology. $\mathrm{He}$ is currently a professor at the Graduate School of Information Sciences, Tohoku University. He is the vice president of Japan Society for Educational Technology (JSET) and a member of the Central Council for Education of Japanese Government. 